\title{
The effectiveness and cost of adopting the Mediterranean diet among British older adults: a brief intervention with two levels of intensity
}

\author{
J. Lara, A. Mackevic, K. Rudgard and J. C. Mathers \\ Human Nutrition Research Centre, Institute for Ageing and Health, Newcastle University, Biomedical Research Building, \\ Campus for Ageing and Vitality. Newcastle Upon Tyne, NE4 5PL, UK
}

There is now strong evidence indicating that a Mediterranean-type diet (MD) comprising higher intakes of vegetables, fruits, legumes, cereals, fish and a moderate intake of red wine during meals, is associated with a lower risk for chronic diseases and improved wellbeing ${ }^{(1)}$. The present pilot study is part of LiveWell, a research programme to develop lifestyle-based interventions to improve healthy ageing in the peri-retirement period.

The aims of the present study were to assess the effectiveness of a brief intervention promoting the Mediterranean diet (MD) with two levels of intensity, and to evaluate the cost of adopting such dietary pattern, among older British adults.

Twenty-three adults $(66 \pm 9 \mathrm{yrs})$ with a mean BMI of $27.1 \pm 5.0$, were randomly assigned to one of two groups in a 3 -week MD intervention. Group 1 attended a single educational group session (EGS) on the benefits of the Mediterranean diet, its principles, shopping and cooking tips; after the EGS, participants were encouraged to adopt the MD for the next 3 weeks. Group 2 attended the EGS and received additional support via telephone as well as additional material (e.g. recipes, menus). Adherence to the MD was assessed before, and 3 weeks after, the EGS. Participants also completed two $3 \mathrm{~d}$ food diaries; one assessed usual diet at baseline, and the second at the end of third week tested for changes in response to the intervention.

A brief intervention promoting the MD was well received. Intensity of this intervention (i.e. Group 1 vs. Group 2) had no significant effect on dietary choices. The group receiving additional support reported better responses to the intervention, however the differences were not statistically significant $(P>0.05)$. Overall, pooled results from all subjects showed that fish intake increased significantly $(p=0.01)$, together with higher but not significant increases in legumes and fruits, between baseline and Week 3.

The mean daily cost of food intake as estimated from 3-day food diaries, was not significantly different between baseline and post intervention ( $£ 5.02 \pm 1.59$ at baseline vs. $£ 5.30 \pm 1.56$ ). These results were in line with participants perceived the cost of adopting the MD. Compared with the perceived cost of their habitual diet at baseline, $75 \%$ of participants reported perceiving the cost of their diet to be the same after the intervention.

In conclusion this brief dietary intervention shows that the MD can be an acceptable, inexpensive and effective approach to healthy eating in the short term among British older adults.

LiveWell is supported by the Lifelong Health and Wellbeing Cross-Council Programme (LLHW). The LLHW funding partners are: Biotechnology and Biological Sciences Research Council, Engineering and Physical Sciences Research Council, Economic and Social Research Council, Medical Research Council, Chief Scientist Office of the Scottish Government Health Directorates, National Institute for Health Research /The Department of Health, The Health and Social Care Research \& Development of the Public Health Agency (Northern Ireland), and Wales Office of Research and Development for Health and Social Care, Welsh Assembly Government.

1. Sofi F, Abbate R, Gensini GF, Casini A (2010) Accruing evidence on benefits of adherence to the Mediterranean diet on health: an updated systematic review and meta-analysis. Am J Clin Nutr 92, 1189-96. 